\title{
Experimental control of Tollmien-Schlichting waves using pressure sensors and plasma actuators
}

\author{
Pedro P. C. Brito ${ }^{1}$, Pierluigi Morra ${ }^{2}$, André V. G. Cavalieri ${ }^{1}$, \\ Tiago B. de Araújo $^{1}$, Dan S. Henningson ${ }^{2}$ and Ardeshir \\ Hanifi $^{2}$ \\ ${ }^{1}$ Instituto Tecnológico de Aeronáutica, Aerodynamics Department, São José dos \\ Campos, 12228900, Brazil \\ ${ }^{2}$ KTH Royal Institute of Technology, Linné FLOW Centre, Dept. of Mechanics, \\ SE-10044, Stockholm, Sweden
}

Internal Report (2020)

This manuscript shows the successful application of the inverse feed-forward control (IFFC) technique for the cancellation of Tollmien-Schlichting (TS) waves. Active wave cancellation of two-dimensional broadband TS disturbances is performed with a single dielectric barrier discharge (DBD) plasma actuator. The measurements required for the IFFC are performed with microphones, instead of hot wires most used for this task, in order to reduce the space occupied by the sensors and assess the suitability of simpler and cheaper devices. The experiments are conducted in an open-circuit wind-tunnel with a NACA0008 wing profile. An attenuation of the TS-wave amplitude of one order of magnitude is achieved. Direct numerical simulations (DNS) are also performed, and compared to the outcome of the experiments. The modeling of both actuator and sensors in the DNS is not based on data from the present experiments. The plasma actuator used is a mapping of the force field in Fabbiane et al., J. Fluid Mech. 2015, to the NACA0008 wing profile, whereas the microphones are modeled as pressure probes. Despite these modelling choices, a remarkable level of agreement between the DNS and the experiments is achieved. However, the control performance is better in the DNS, with attenuations of three orders of magnitude of TS-wave amplitude. Further analysis of experiments and simulations shows that the limiting factor in the experiments is the ambient low-frequency acoustic waves in the wind tunnel, which are sensed by the microphones, acting as noise in the analysis of TS-wave evolution and thus leading to lower coherences between sensors and actuators. This in turn leads to a suboptimal control kernel in the experiment. 


\section{Introduction}

The delay of laminar to turbulence transition in a boundary layer flow results in a reduction of the friction drag, which implies less energy consumption for many applications, such as transportation means like aircrafts. Therefore, control methods focused on maintaining the boundary-layer flow in a laminar state are of great interest. The mechanisms behind laminar-turbulent transition in a boundary-layer flow are different and depend on a number of parameters; an overall picture of these different scenarios can be found in Schmid \& Henningson (2001). In two-dimensional flows, when the intensity of the background disturbance is very low, $\approx 0.05 \%$ of the free-stream velocity, transition to turbulence is caused by Tollmien-Schlichting (TS) waves. These are packets of travelling waves with small streamwise scales and relatively large spanwise extent, whose amplitude grows exponentially as the wave packet is advected downstream. When the amplitude of TS-waves reaches a few percent of the free-stream velocity the wave-packet breaks down and causes transition to turbulence. The aim of most of the control strategies to delay transition to turbulence is damping the growth of the TS-waves before the break-down.

The energy growth of TS-waves is governed by a linear mechanism. This characteristic is usually exploited to choose the technique to dampen the amplification of the TS-waves. Thomas (1983) and Laurien \& Kleiser (1989) made use of this a priori knowledge to attenuate TS waves and delay transition. In some first attempts to achieve flow control the linearized Navier-Stokes system was used to create ad hoc counter disturbances. Such a practice lacks in generality and may need tedious testing. Thus, the application of control theory to flow control problems is now common, of which Högberg et al. (2003), Sipp et al. (2010), Kotsonis et al. (2013), Hanson et al. (2014), Fabbiane et al. (2015), Simon et al. (2016), Morra et al. (2020) are examples. Optimal control methods aim at computing the function that provides the actuation signal from measurements while minimizing an objective function. A commonly used control technique is the linear quadratic Gaussian regulator (LQG), whose objective function is based on the 2-norm of a chosen output and which assumes the system to be linear and driven by stochastic disturbances, with the measurements corrupted by white noise (Lewis \& Syrmos 1995). LQG is based on a state-space description of the system, assumes the relationship between the outputs and the inputs as linear, and gives the optimal solution by solving an algebraic Riccati equation. The Riccati equation consists of a matrix equation whose dimensions are approximately those of the linear system to control. If the dimension of the system is large, as it is in fluid mechanics, the solution of the Riccati equation can be computationally demanding. Thus, reduced order models (ROM) are usually employed for a reduce-then-control design approach (Anderson \& Liu 1989). Another option to avoid demanding computations for solving the control problem is discussed by Fabbiane et al. (2014), who employed a control technique that only requires the modelling of the transfer function ( $\mathrm{TF}$ ) from the actuators to the measurements. 
Among the methods to build a balance-truncated ROM using proper orthogonal decomposition (POD) is the most spread one in literature for its reliability and performance (Rowley \& Dawson 2017; Barbagallo et al. 2009; Bagheri et al. 2009; Ilak \& Rowley 2008). Balanced POD truncation can be attained with a snapshot-based method (Rowley 2005), or by the eigensystem realization algorithm (ERA) (Ma et al. 2011; Juang \& Pappa 1985). The snapshot-based method is usually computationally more demanding than the ERA because it requires the explicit computation of the modes from the Navier-Stokes and its adjoint system whereas ERA only requires input-output time signals, which makes ERA suitable in wind-tunnel experiments. Ma et al. (2011) proved that the ROM obtained with ERA-POD is formally equivalent to snapshot-based balanced POD truncation. Both snapshot based balanced POD truncation and ERA-POD are based on the impulse responses of the system; examples of the successful application of ERA-POD are Semeraro et al. (2013), Sasaki et al. (2018a), Morra et al. (2020).

It is not possible to produce a perfect impulse input with an experimental apparatus, instead a finite time pulse is used. However, in many systems a very short and strong pulse may drive the actuator to off-design working conditions and result in unwanted responses. In these cases the actuator is driven with a pseudo-random time signal and the impulse response is computed as an estimate of the input-output TF, which is by definition equivalent to the impulse response. There are many techniques for system identification of linear dynamical systems (see Ljung (1999) for a survey), among which one of the simplest to implement yet very effective is TF estimation via power spectra density estimation with the Welch method (Stoica \& Moses 2007). A combination of this estimation technique with ERA is presented in Morra et al. (2020).

Flow control techniques are usually referred to as passive or active. Passive control techniques modify the mean flow through surface shaping or passive devices (e.g. vortex generators) such that growth of perturbations is suppressed. Whereas, active control techniques can be used to generate counteracting disturbances by means of an external energy source. This energy source is regulated by a signal whose amplitude is a function of time. Flow control techniques where this time signal is constant (Gad-el Hak 2000) prove to be unsustainable because they require high actuation energy. This occurs because these control methods aim at modifying the baseflow to dampen the disturbances. On the other hand, reactive flow control techniques, where the time signal is not constant, appear to require less actuation energy because they act directly on the perturbation. Reactive flow control techniques with moving wall actuators showed to successfully dampen TS-waves both in wind-tunnel experiments (Milling 1981; Liepmann \& Nosenchuck 1982,?; Thomas 1983) and in in-flight tests (Sturzebecher \& Nitsche 2003).

The choice of the actuator is critical in flow control applications (Cattafesta \& Sheplak 2010; Sasaki et al. 2020) because it defines the type of disturbance that can be canceled. Using actuators with mechanical parts can be a limitation 
in certain applications, so some studies focus on actuators without mechanical parts. Among them, the plasma actuator has been studied extensively (Corke et al. 2010; Kriegseis et al. 2011; Kriegseis 2011; Duchmann et al. 2014; Grundmann et al. 2014; Jolibois \& Moreau 2009), and is now known to be able to attenuate TS-waves (Grundmann \& Tropea 2008; Kurz et al. 2013, 2014; Kotsonis et al. 2013, 2015; Duchmann et al. 2014; Fabbiane et al. 2015; Simon et al. 2015, 2016). A comprehensive review on the advantages and disadvantages of employing the plasma actuators as a flow control device is given in Wang et al. (2013) and Kriegseis et al. (2016).

The type of sensor is also a critical design choice because it defines the operators that extract the output from the system dynamics. Most of the experimental studies to investigate laminar-turbulent transition delay employ hot wire anemometry or hot film sensors to obtain velocity measurements or shear measurements. Although these sensors prove to be effective (Sturzebecher \& Nitsche 2003; Fabbiane et al. 2015; Simon et al. 2015), their adoption in an application may be problematic. Hot wires are invasive and the wires break with ease. Wall shear-stress sensors are not invasive because they are located on the wall, but they are not robust to temperature fluctuations, uncertainties are difficult to quantify (Sheplak et al. 2004), and usually have a low signalto-noise ratio. Instead, pressure measurements (e.g. microphones) usually are easier to handle and have high signal-to-noise ratio. Moreover, microphones can be cheaper and more robust than hot-wires or wall shear-stress sensors, which allow their use in higher quantities to sense more disturbances such as three-dimensional TS waves..

The position of sensors and actuators is another key aspect in control problems. For convectively unstable flows, as boundary layer flows, the setup that can reach the best nominal performance and guarantees robust stability is the feedforward configuration (Devasia 2002; Belson et al. 2013; Sipp \& Schmid 2016; Schmid \& Sipp 2016). Fabbiane et al. (2015) highlighted the performance issues and robustness properties of a model-based LQG controller in a feedforward configuration for the boundary layer flow with zero pressure gradient over a flat plate. Moreover, Fabbiane et al. (2015) employed plasma actuators and hot-wire velocity measurements and demonstrated, for the first time, TS-wave attenuation in wind-tunnel experiments using 2-D model-based LQG compensators without any model fitting or system identification. If the control system is in a feedforward configuration, the inverse feedforward control (IFFC) tehcnique can be applied. IFFC is an optimal control technique which is less computationally demanding than LQG because does not require the solution of an algebraic Riccati equation and bypasses the computation of a state-space ROM. In fact, IFFC is based solely on the input-output representation of the system dynamics, which coincides with a TF representation. Such TFs can be directly extracted from experiments using the Welch method, as discussed above. The objective function of IFFC is the 2-norm of a chosen output. In Sasaki et al. (2018a) and Morra et al. (2020) it is shown with direct numerical simulations (DNS) that IFFC is effective for disturbance attenuation 
of TS-waves and free-stream induced streaky structures.

The present work presents the successful application of IFFC technique in a wind-tunnel experiment and DNS for the cancellation of TS waves over a wing profile. Active wave cancellation of two-dimensional broadband TS disturbances is performed with a single dielectric barrier discharge (DBD) plasma actuator. The measurements required for the IFFC are performed with microphones instead of hot-wires to reduce the space occupied by the sensors and assess the suitability of cheaper devices. The experiments are conducted in an open-circuit wind-tunnel with a NACA0008 wing profile. DNS for similar geometry and flow conditions are performed and compared to the outcome of the experiments. DNS data are further used to explain differences in performance of control in experiments and simulations.

\section{Control methods}

\subsection{Inverse Feed-Forward Control}

Given a linear dynamical system with disturbance input $d(t)$, actuation input $u(t)$ and outputs $y(t)$ and $z(t)$, the frequency domain TF representation can be written as

$$
\begin{aligned}
& \hat{y}(\omega)=\hat{G}^{d y}(\omega) \hat{d}(\omega), \\
& \hat{z}(\omega)=\hat{G}^{u z}(\omega) \hat{u}(\omega)+\hat{G}^{d z}(\omega) \hat{d}(\omega),
\end{aligned}
$$

with the representing the Fourier transformed quantity, and $\hat{G}$ the input-output transfer function. Here, it is assumed that the input $u$ does not influence the output $y$, which is generally the case for convectively unstable flows with output $y$ placed upstream of actuator $u$. The relationship between the outputs $y$ and $z$ can be written as

$$
\hat{z}=\hat{G}^{y z} \hat{y}+\hat{r},
$$

where $\hat{r}$ is a residual part of $\hat{z}$ which cannot be retrieved by the output $\hat{y}$ (i.e. external inputs not measured by $y$ ), and $\hat{r}$ is unknown. Combining equations $1 a, b$ and 2 and neglecting the unknown term $\hat{r}$ allows computing the input $u$ based on $y$ that reduces the most the amplitude of $z$ (complete cancelation is possible only if $\hat{r}$ is known or equal to zero). This is the idea behind inverse feed-forward control. However, this procedure leads to a result ill conditioned in the zeros of $\hat{G}^{u y}$, which can result in spurious high amplitudes of the input. Moreover, model uncertainties are not considered, and unstable zeros in $\hat{G}^{u z}$ may lead to an unstable controller. Such limitations are addressed in Devasia (2002).

Assume $\hat{u}=\hat{K} \hat{y}$. The technique proposed in Devasia (2002) aims at computing $\hat{K}$ by minimizing the objective functional

$$
J=\int_{-\infty}^{\infty} \hat{u}^{H} \hat{R} \hat{u}+\hat{z}^{H} \hat{Q} \hat{z} \mathrm{~d} \omega
$$


with ${ }^{H}$ the complex conjugate, and $\hat{R}$ and $\hat{Q}$ user-defined design weights. The presence of the objective functional changes the nature of the problem into an $\mathcal{H}_{2}$ optimal control problem, whose solution is given by

$$
\hat{K}=\frac{\left(\hat{G}^{u z}\right)^{H} \hat{Q} \hat{G}^{y z}}{\hat{R}+\left(\hat{G}^{u z}\right)^{H} \hat{Q} \hat{G}^{u z}} .
$$

In this work, $\hat{R}$ and $\hat{Q}$ are always taken as constants. Moreover, for the present setup, with a single actuation $u$ which is a function of a single sensor $y, \hat{Q}$ and $\hat{R}$ are scalars, and the kernel only depends on the ratio between them. Accordingly, we kept $\hat{Q}=1$ and varied $\hat{R}$ aiming at maximizing the closed-loop performance. This technique was successfully applied in DNS to damp streaky structures and TS-waves in the Blasius boundary layer flow over a flat plate (Sasaki et al. 2018a; Morra et al. 2020).

The inverse Fourier transform of the control law $\hat{u}(\omega)=\hat{K}(\omega) \hat{y}(\omega)$ leads to

$$
u(t)=\int_{-\infty}^{\infty} K(\tau) y(t-\tau) \mathrm{d} \tau
$$

a convolution integral which in real time can only be carried out for positive time delay $\tau$. The lower integration limit is thus taken at $\tau=0$ and the integral is discretised, such that

$$
u(t)=\sum_{n=0}^{N} K(n \Delta t) y(t-n \Delta t) .
$$

Notice that this corresponds to truncating the control kernel $K(\tau)$ to its causal part. When the actuation is placed downstream of the sensor $y$, the kernel is seen to be indeed causal (Sasaki et al. 2018a,b), and such truncation has no effect on control performance. However, if $K(\tau)$ remains significant for $\tau<0$ such truncation leads to a suboptimal control law. This issue is found to be relevant in the present studies.

\subsection{TF Identification}

Equation (4) shows that $\hat{G}^{y z}$ and $\hat{G}^{u z}$ are necessary to compute the controller. An effective method to compute these TFs is estimating them from the time series of the signals. $\hat{G}^{y z}$ is estimated from the outputs $y$ and $z$, while $\hat{G}^{u z}$ is estimated from the input $u$ and the output $z$. It can be shown (Bendat \& Piersol 2011) that the optimal frequency response, in the least square sense, is achieved with

$$
\hat{G}^{y z}=\frac{\hat{S}^{y z}}{\hat{S}^{y y}}, \quad \hat{G}^{u z}=\frac{\hat{S}^{u z}}{\hat{S}^{u u}}
$$

where $\hat{S}^{y y}$ and $\hat{S}^{y y}$ are the power spectral densities of the signals $u$ and $y$, whereas $\hat{S}^{y z}$ and $\hat{S}^{u z}$ are the cross-spectral densities of the signals $u, y$, and $z$. Since the time signals available are over a finite time interval it is not possible to compute the spectral densities exactly, so an estimation method is employed. Here, the spectral densities are estimated with the Welch method (Stoica \& 
Moses 2007). The method consists in splitting the available time signal in sub-intervals, multiplying each sub-interval with a window function to reduce spectral leakage, evaluating the Fourier Transform for each sub-interval, and average the amplitude of each frequency over the set of sub-intervals. Here, the window function used is the Hamming window. Equation (7), sometimes referred to as an $H^{1}$ estimator (Rocklin et al. 1985), minimizes the error due to noise in the output.

\section{Plant}

\subsection{Experimental Set-up}

Experiments were carried at Instituto Tecnológico de Aeronáutica (ITA) at the Kwei Lien Feng laboratory in an open-circuit wind tunnel capable of reaching velocities up to $80 \mathrm{~m} / \mathrm{s}$. The test section has $4.0 \mathrm{~m} \times 1.28 \mathrm{~m} \times 1.0 \mathrm{~m}$ and the turbulence level is less than $0.1 \%$. The experiments were performed for a chordbased Reynolds number of $5.33 \times 10^{5}$ with a NACA0008 airfoil manufactured by SAAB within the framework of the SweDemo project funded by the Swedish national aeronautical program (NFFP). The original model has $0.8 \mathrm{~m}$ of chord and $0.5 \mathrm{~m}$ of span. The airfoil was made of composite materials and had a plexiglass plate mounted at the center to allow easy assembling of different sensors and actuators. Besides the main airfoil two similar sections of $0.25 \mathrm{~m}$ of span were manufactured in wood to fill the $0.5 \mathrm{~m}$ gap and fit the model in the test section of the wind tunnel. A slit situated at $x / c=0.1$ was connected, with tubes of equal length, to a loudspeaker outside the wind tunnel, which was used to trigger the TS waves. The plasma actuator is located at $x / c=0.33$. Figure 1 shows the model placed in the test section.

In this work we investigate the feasibility of closed-loop flow control with such microphones. The advantages of using microphones rely on the fact that they are less intrusive than typical hot wires, as they are placed tangent to the wall. Perhaps more importantly, electret microphones have a very low cost, which makes them suitable for flight test campaigns and situations were a large number of sensors need to be placed. The microphones had a diameter of $6.35 \times 10^{-3} \mathrm{~m}$ and were positioned at $x / c=0.3$ and $x / c=0.4$.

The acquisition system used was a DS1104 R\&D Controller Board manufactured by dSPACER. It is a real time processor board, coupled to a computer

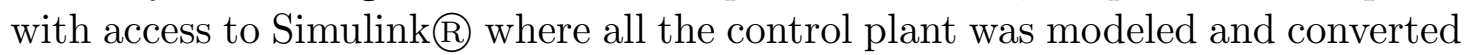
to the DS1104 format. The working frequency was $10 \mathrm{kHz}$. The input (ADC) and output (DAC) communications were performed using BNC connectors and the ControlDesk interface, which allows real-time modification of the input values of the plant.

A Minipuls 2.1 system, manufactured by GBS Elektronik @, was used as the high voltage source to generate plasma. The system generates $\mathrm{AC}$ voltage up to $20 \mathrm{kV}(\mathrm{Vpp})$ with an operational frequency range of $5-20 \mathrm{kHz}$. The device is mainly a bridge converter and a transformer cascade that was connected to 
a power supply unit that provided an input of $23 \mathrm{~V}$. The system provided a potential difference around $10 \mathrm{kV}$ at the dielectric (plexiglass plate) throughout two thin strips of copper that worked as electrodes along the span of the wing, one electrode placed outside of the wing and the other placed inside. Plasma was generated at both sides of the plexiglass plate; however, a plastic tape was used to cover the inner side copper electrode in order to allow plasma only on the wing surface. In order to monitor the proper operation of the Minipuls platform, an oscilloscope was used to visualize if the wave shape was consistent with the input signals from the ControlDesk of the DS1104 and to check the output voltage.

A Vishayß signal conditioner was used before sending the signals to the DS1104 platform. An NCA® amplifier of $8 \mathrm{Ohms}$ was also used to send the perturbation signal (white noise) generated by the plant and controlled by the ControlDesk interface. The amplifier sent the signal to a large speaker of $200 \mathrm{~W}$ which propagated the perturbation sound through 37 tubes radially distributed and all connected to the slit on the wing surface.

The experimental set up involves the determination of parameters before running the experiment successfully. These parameters are the offset level and amplitude for modulation of the plasma and the amplitude level for the loudspeaker.

The offset level of the plasma goes from $1.2 \mathrm{~V}$ to $1.9 \mathrm{~V}$, corresponding to $0 \%$ and $100 \%$ power, respectively. The offset level is one of the main parameters that dictate the energy spent to perform the attenuation of the TS waves, so it is desired to perform the control with the minimum offset level as possible. The offset level of the plasma required to perform the experiments is mainly affected by the humidity of the air, for humid air higher offsets are required to generate stable plasma. For the present results, the humidity of the air was around $35 \%$ and a low offset level of $1.25 \mathrm{~V}$ as found to be sufficient. This was determined by verifying that application of plasma with this offset without amplitude modulation leads to slight reductions of TS-wave amplitude measured by the $z$ microphone, which ensures that plasma is generated by the actuator, without excessive power.

A second parameter is the amplitude of plasma modulation while acquiring the signals in open-loop to compute the transfer functions required to compute the control kernels. This amplitude affects the level of coherence between $u$ and $z$ signals. Too small amplitudes results in low coherence due to low sensitivity of microphones and high amplitudes gives low coherence due to introduced non-linearities. An amplitude of $0.05 \mathrm{~V}$ around the offset level $(1.25 \mathrm{~V})$ in the open-loop control stage resulted in the highest mean value for magnitude-squared coherence between $u$ and $z$, about 0.85 .

Similarly, the loudspeaker also required an amplitude to restrict the white noise level. Experimental tests were performed to define the limit where nonlinearities started to appear. This was done by the PSD of the signals in the frequency range of TS waves for different amplitude levels. The amplitude level 


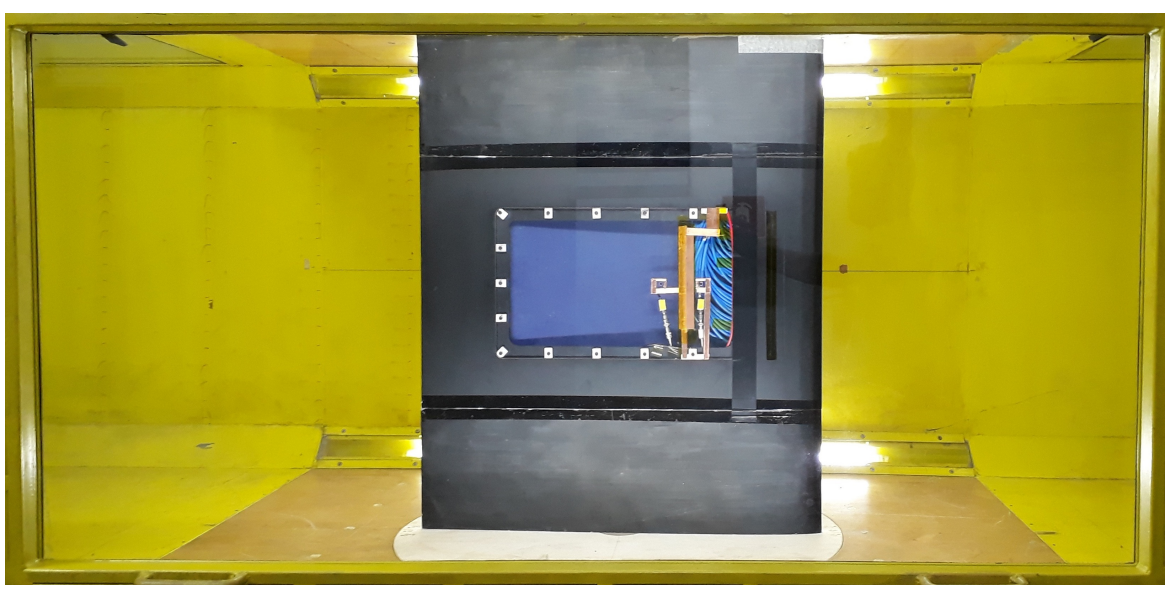

Figure 1: Side view of the test section.

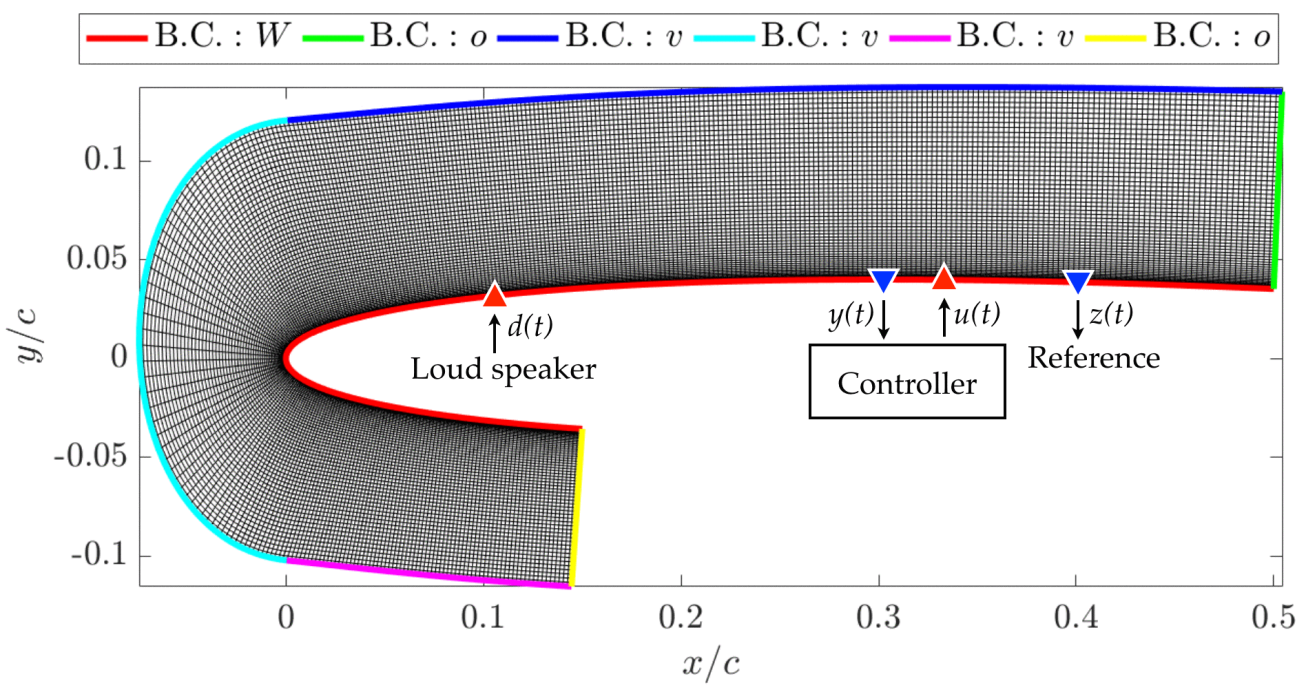

Figure 2: Nek5000 grid. Boundary Condition(BC), W: wall, v: Dirichlet, o: outflow.

was chosen to ensure the maximal coherence level between $d$ to $y$ and $z$, which is between $90 \%$ and $95 \%$ for both sensors, as shown in figure 5 .

\subsection{Numerical Set-up}

The direct numerical simulations (DNS) of the linearized Navier-Stokes equations are performed on a domain which is consistent with the experimental set-up. DNS are performed using Nek5000 (Fischer et al. 2008). Since only twodimensional TS waves are considered in this work, the computational domain is two-dimensional. Moreover, since performing a DNS of a domain which includes the whole test-section is computationally demanding, only a portion of this domain is simulated. This subdomain is chosen such that sensors, actuators and 
the boundary-layer are included, as shown in figure 2. The boundary conditions are: no-slip on the wing surface, Dirichlet on outer boundary and stress-free on outflow boundaries. The Dirichlet BCs are extracted from the results of laminar Navier-Stokes computations including the whole test-section of the wind tunnel. The Navier-Stokes computations are performed in ANSYS Fluent. In both numerical simulations the chord-based Reynolds number was $R e_{c}=533000$ matching the experiments. The initial disturbances are generated by as a Gaussian distributed wall-normal forcing centered on the wall at $x / c=0.1$, which mimics the effect of the speaker used in the experiment. The sensors are modeled to compute the average value of the pressure over a finite surface in order to mimic the microphones used in the experiments, and they are located at $x / c=0.3$ and $x / c=0.4$. The actuator is modeled as a streamwise forcing, whose spatial distribution is in accordance with the results from Kriegseis et al. (2013). The controller $\mathrm{K}(\mathrm{t})$ obtained from the DNS data is used in input-output simulations based on signals only, similarly to the procedure in Morra et al. (2020).

\section{Results}

\subsection{Spectra and input-output behaviour}

The DNS does not include any ad-hoc modeling to describe some specific characteristics of the wind-tunnel or devices used in the experiments. Sensors and actuators are modeled to mimic the operations of the real devices but are not built to fit the experimental set-up. Moreover, experimental data present a non-negligible amount of noise, which is not modeled in the numerical simulations.

The input-output transfer functions obtained from the signals collected in the DNS are based on impulse response simulations. This allows avoiding the application of the Welch's method to estimate the PSD, so the errors introduced by construction in the estimation are not present. Figure 3 shows the PSDs of both output signals, $y$ and $z$, collected in the DNS from stochastic input disturbance with those of the signals collected in the experiments; in this case both are computed using Welch's method. The amplitudes are rescaled in the same way that the DNS are made. It is clear that the DNS reproduces reasonably the evolution of the disturbances in the boundary layer in a neighborhood of $f=200 \mathrm{~Hz}$, which is close to the peak frequency of TS waves for the present flow, while a different trend appears for $f>400 \mathrm{~Hz}$, which is likely due to high-frequency noise in the experiment. A different behavior is also noticed for $f<100 \mathrm{~Hz}$. These differences are thought to be noise due to the acoustics of the wind tunnel, mainly due to the fan noise which reverberates in the section, and because of electromagnetic noise due to the electrical networking which causes a salient peak around $60 \mathrm{~Hz}$.

Regardless the noise implicit in the experimental data, the frequency range in which the TS waves are amplified in experiments and DNS, $150 \mathrm{~Hz}<f<350 \mathrm{~Hz}$,

show to be in good agreement, especially for the $\hat{S}_{y y}$ spectrum of the upstream 

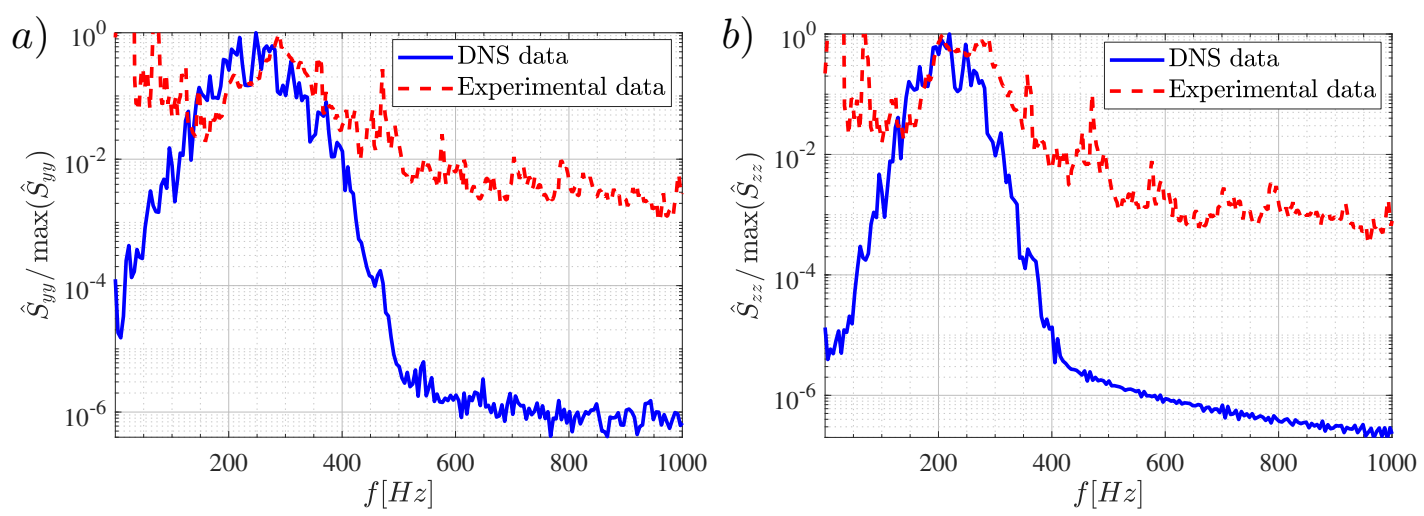

Figure 3: PSD of $y$ (left) and $z$ (right) outputs. Comparison of DNS vs experimental data.
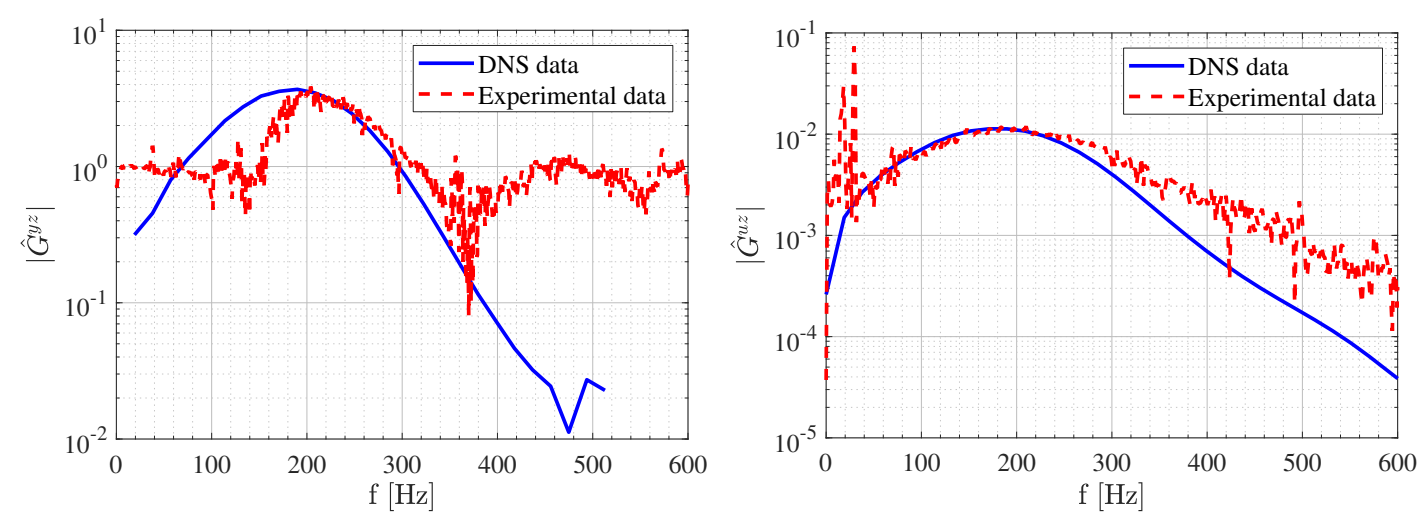

Figure 4: Left: transfer function $\hat{G}^{y z}$ comparison in frequency domain: DNS vs experimental data. Right: transfer function $\hat{G}^{u z}$ comparison in frequency domain: DNS vs experimental data.

microphone.

Figures 4 shows a comparison of the transfer functions identified via the DNS and the experimental results. The transfer functions based on the experimental data are less smooth than the one based on the DNS data due to the ambient noise levels. Despite this issue, the shape of the transfer functions is in fairly good agreement for both cases. The exception, is the experimental frequencies below $150 \mathrm{~Hz}$ for the $\hat{G}^{y z}$ where the experimental data presents a gap compared to the DNS data. This discrepancy can be explained with the results of figure 5, which shows the coherence between loudspeaker excitation and measured pressure, showing that signals for frequencies below $150 \mathrm{~Hz}$ are not related to the TSwave excitation. Frequencies lower than $150 \mathrm{~Hz}$ and higher than $400 \mathrm{~Hz}$ in the experiment are thus incoherent with the upstream excitation, and are likely related to the acoustic waves inside the test section, as discussed before.

Figure 6 shows the transfer functions $G^{y z}$ and $G^{u z}$ in time domain where 

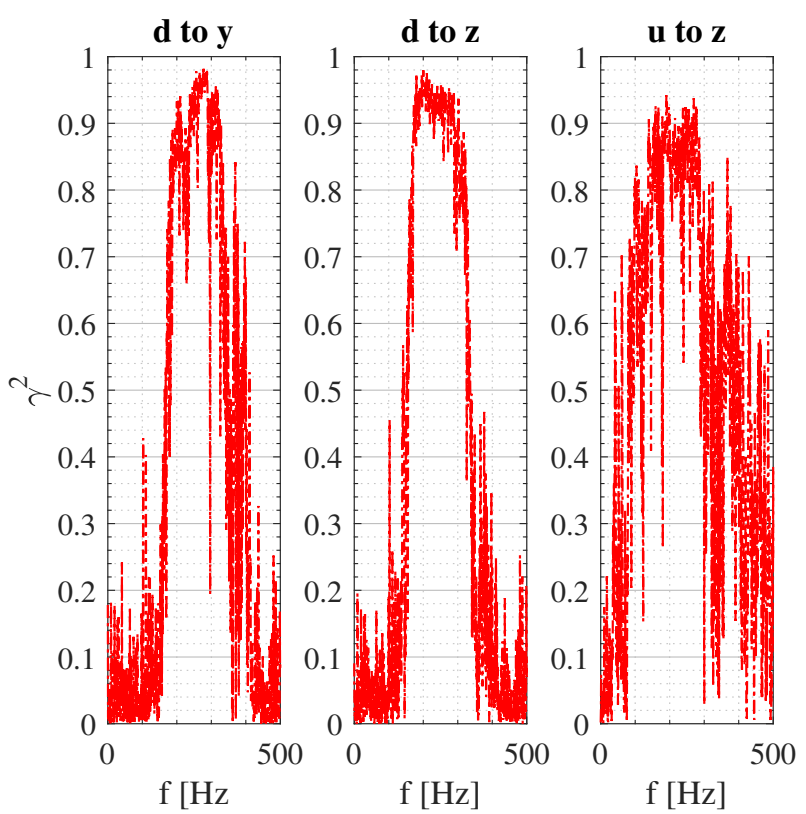

Figure 5: Experimental coherence values between signals from $d$ to $y$ (left), from $d$ to $z$ (middle), and from $u$ to $z$.
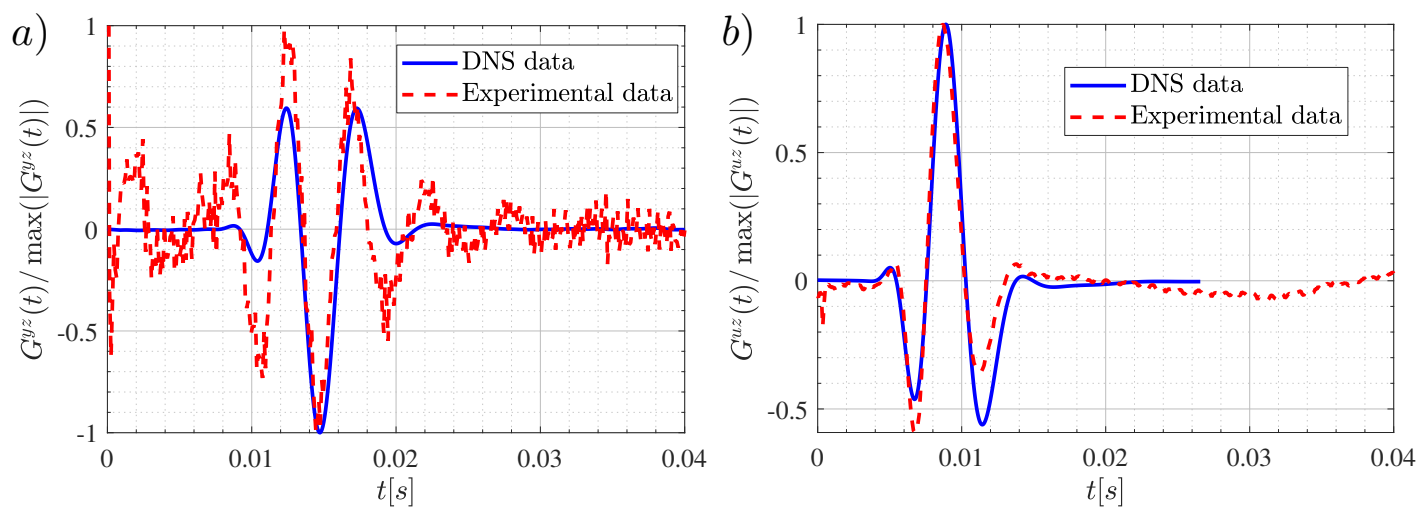

Figure 6: Transfer functions comparison: DNS vs experimental data. $a): G_{y z}(t)$. $b): G_{u z}(t)$; there is not time delay for this transfer function.

the expected good agreement is confirmed and the presence of noise for low and high frequencies is easily identified in $G^{y z}$. We also observe that since experimental $\hat{G}^{y z}$ is narrower in the frequency-domain (figure 5), due to the coherence drop below $150 \mathrm{~Hz}$, its time-domain counterpart $G^{y z}$ is more spread than the numerical data. This issue is relevant for the control kernels, which are shown in figure 7 . The differences in the frequency content of the transfer functions from experimental and DNS data are the reason for which the kernels in figure 7 are different. The fact that $\hat{G}^{y z}$ from experimental data is narrower than $\hat{G}^{y z}$ from DNS data, as shown in figure 4, causes the kernel from experimental 


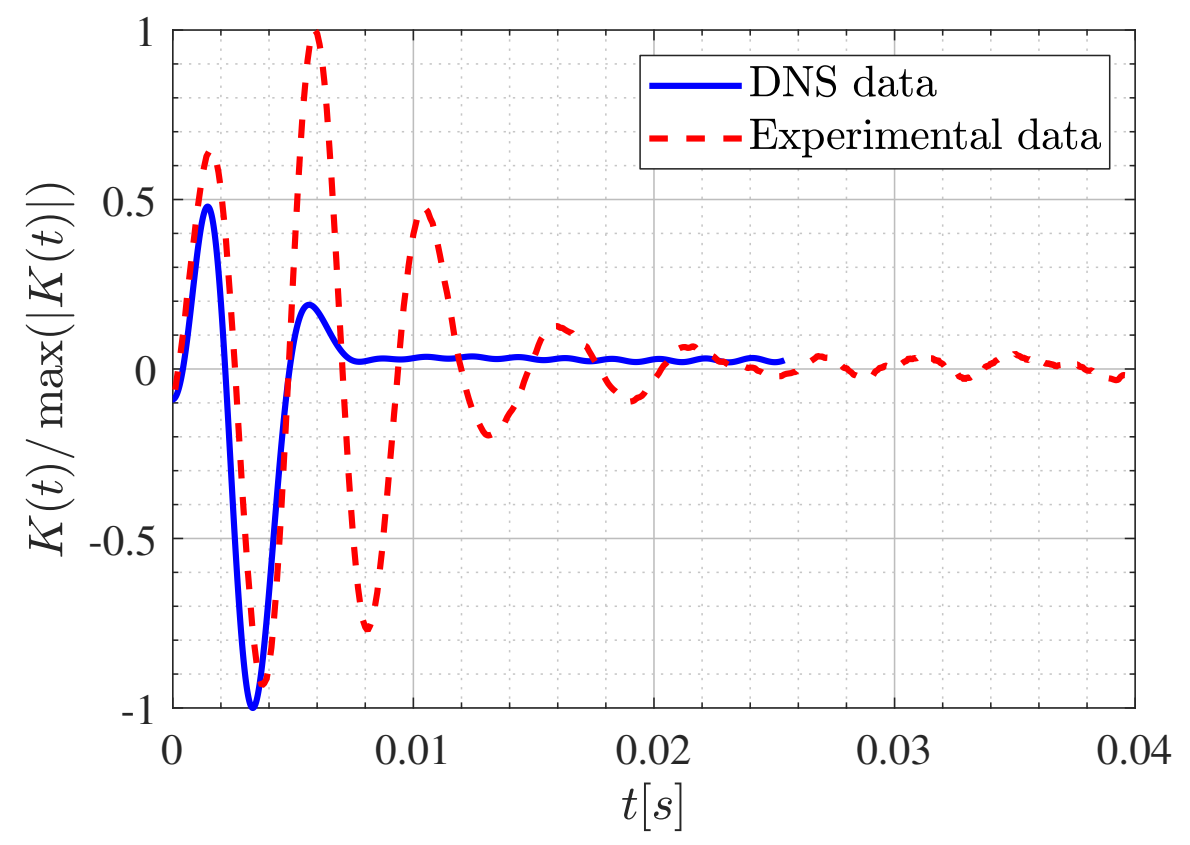

Figure 7: Comparison of the transfer functions of the controller $K(t)$ correspongin to $\mathrm{R}=200$. DNS vs experimental data.

data to be less localized than the kernel from DNS data. This occurs because $G^{y z}$ is at the numerator to compute $\hat{K}$ in equation (4). The presence of noise at $f>350 \mathrm{~Hz}$ in $\hat{G}^{y z}$ is attenuated in the computation of $\hat{K}$ because of the multiplication with $\hat{G}^{u z}$, which decays in the same frequency range as shown in figure 4 (right). The decay of $\hat{G}^{u z}$ is fast enough to compensate the noise content in $\hat{G}^{y z}$ and bring it to a negligible level. This is the reason for which the kernel from experimental data does not show high frequency oscillations in figure 7 .

\subsection{Closed-loop control}

Besides the transfer functions, the determination of the control kernels requires a specification of the penalization factor $R$. Here, it was left to be tested experimentally by evaluating the control performance. During the experiment different $R$ values between 100 and 400 were tested. Figure 8 illustrates how the different $\mathrm{R}$ affects the actuation, and it is possible to observe how insufficient penalization might lead to excessive actuation signals. For the penalization $R=100$ the actuation crosses the plasma lower operational limit $(1.2 \mathrm{~V})$ which would turn off plasma. Moreover, around $t=0.018$ seconds a high amplitude is noticed, which can also lead to excessive actuation signals.

A clearer view of the effect of the penalization factor can be seen in the closed-loop control results. Figures 9a,b,c show the average PSD for six set of data $(\mathrm{N}=6)$ acquired by the $z$ pressure sensor for three different conditions: TS waves excited by the loudspeaker but no plasma actuation (Plasma Off), 


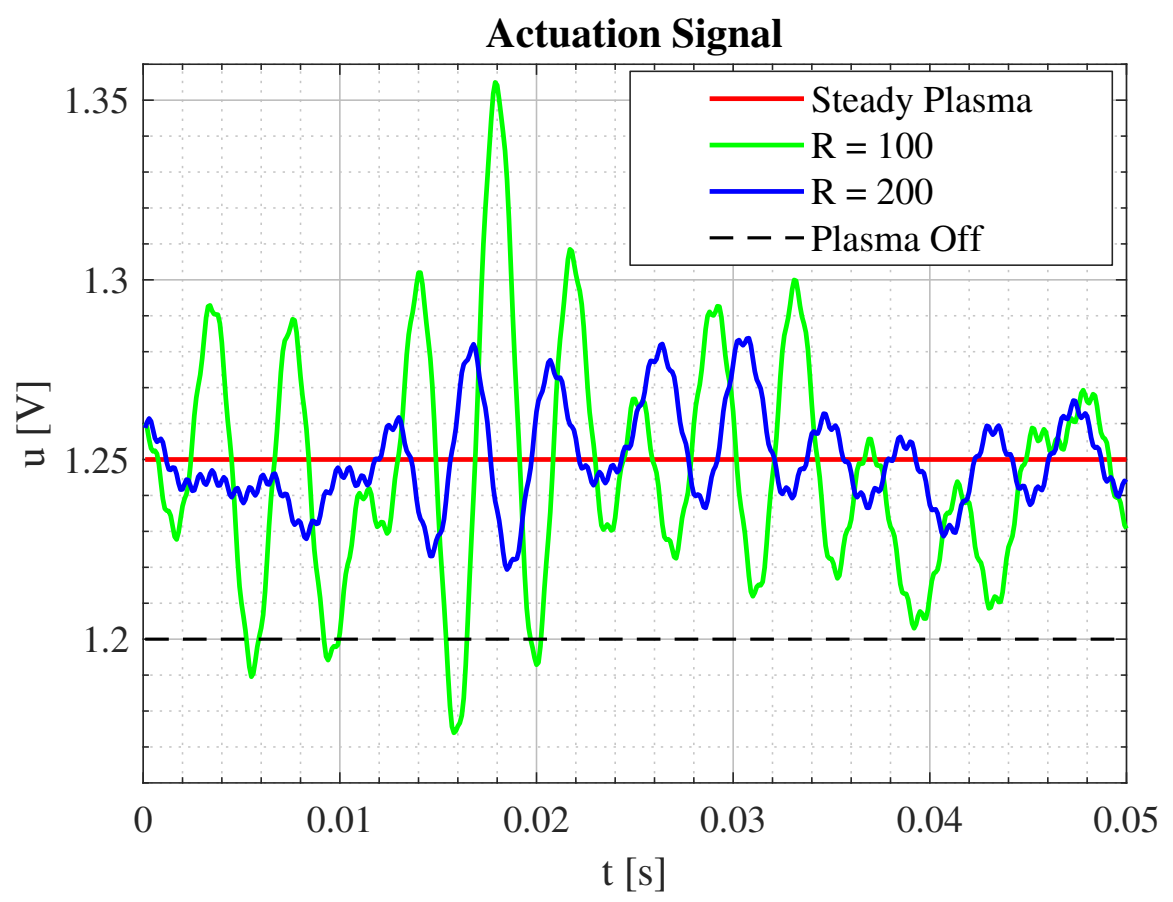

Figure 8: Actuation signals $u$ for steady plasma and for modulated one with $\mathrm{R}=100$ and $\mathrm{R}=200$.

when the plasma is turned on with no modulation (Steady Plasma) and the cases in which the closed-loop control is performed (IFFC). The figures show the average control performance, and also display standard deviations of the PSD (labelled as STD), computed based on 6 separate control experiments. The non-dimensional frequency $F=\left(2 \pi \nu / U_{\infty}^{2}\right) f$ is reported at the upper axis of Figures 9a,b,c.

Figure 9a shows how a closed-loop control signal with insufficient penalization leads to an attenuation worse than the steady plasma case for the lower frequencies, with modest benefits for frequencies of about $250 \mathrm{~Hz}$ and higher. Increasing the penalization to $R=200$ (figure $9 \mathrm{~b}$ ) results in a much better result for the range of frequencies that contains the TS waves. When larger values of $R$ are applied the performance of the system tends to deteriorate, as high penalizations decrease the actuation amplitudes, reducing the amplitude of counteracting TS waves. This trend can be seen in figure $9 \mathrm{c}$ with the blue curve showing the IFFC result starting to shift back to the steady plasma condition.

Figures 9a,b,c also show a considerable standard deviation (STD) for the IFFC control responses. This behavior is mainly due to the fluctuation in the freestream velocity of the wind tunnel, which can be higher than $0.5 \mathrm{~m} / \mathrm{s}$. When the velocity in the test section is close to the target velocity, for which the kernels were computed, the performance is near the one described by the lower standard deviation curve. It is known that TS-wave control performance deteriorates for off-design freestream velocities (Fabbiane et al. 2015). This 

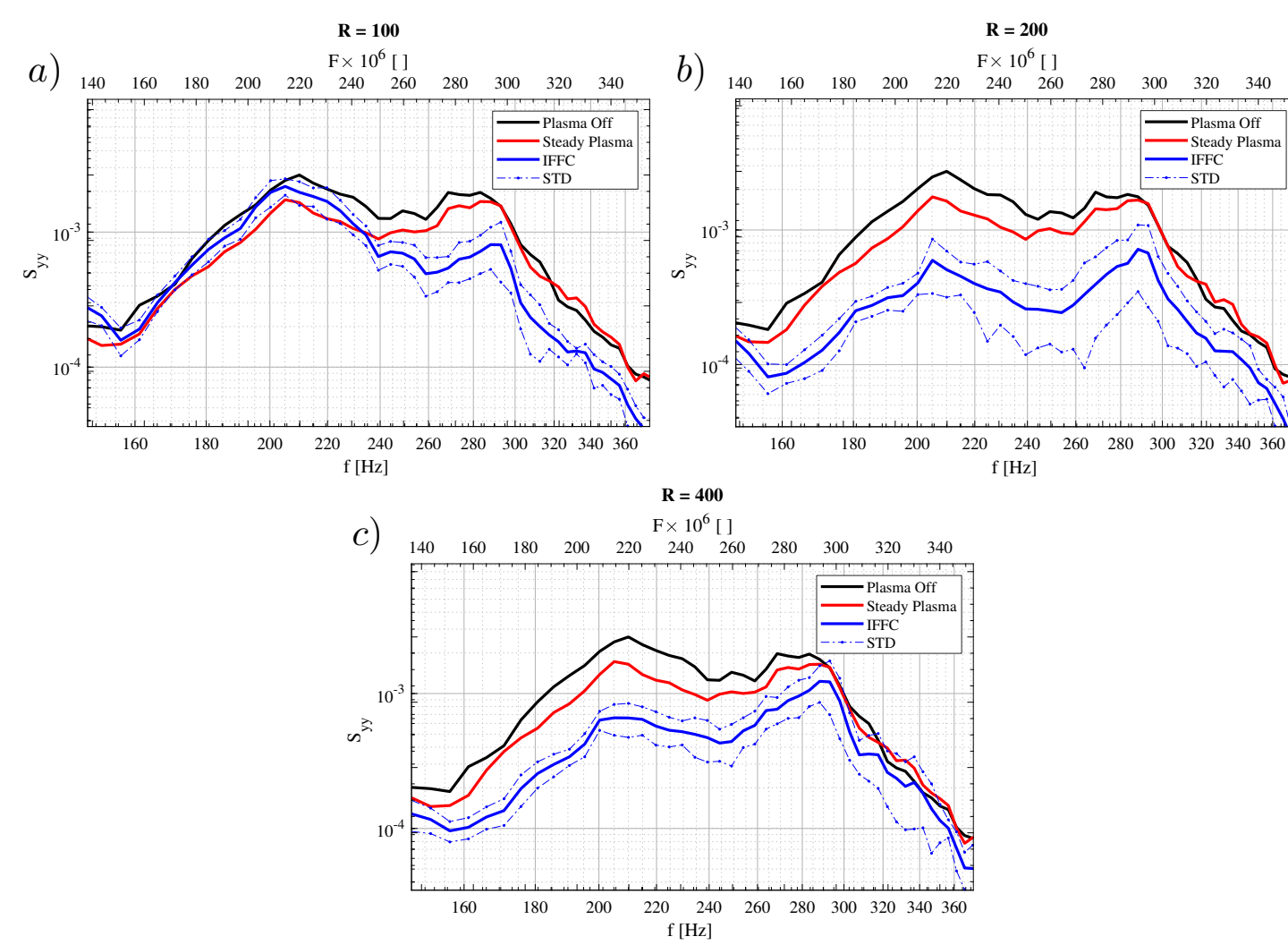

Figure 9: PSD of $z$ pressure sensor. a) $R=100$. b) $R=200$. c) $R=400$.

issue may be circumvented using adaptive control, or a gain scheduling scheme to change kernels if the freestream velocity is varied. It must be mentioned that the fluctuation problem is also implicit in the control plant, because it cannot be guaranteed that the long time signals required to computed the kernel were acquired at a constant velocity. We have nonetheless obtained reductions in power spectra close to one order of magnitude, which shows the potential of IFFC for practical applications of transition control.

\subsection{Comparison of experimental and theoretical control performances}

Now we compare performance of our control approach in experiments and in simulations and try to identify performance limitations in the experiments. Figure 10 (left) shows a comparison between the computed performance and the one obtained experimentally. The computed performance was determined using half of the experimental data to calculate the transfer functions and the other half to compute the two performance curves shown in figure 10 (left). This way the performance are not tested on the same set data on which the coefficients of the transfer functions are fit. The expected performance was determined using an inexpensive approach based on convolution, so that several penalization levels could be easily tested. Equation 8 shows how we can estimate the output 

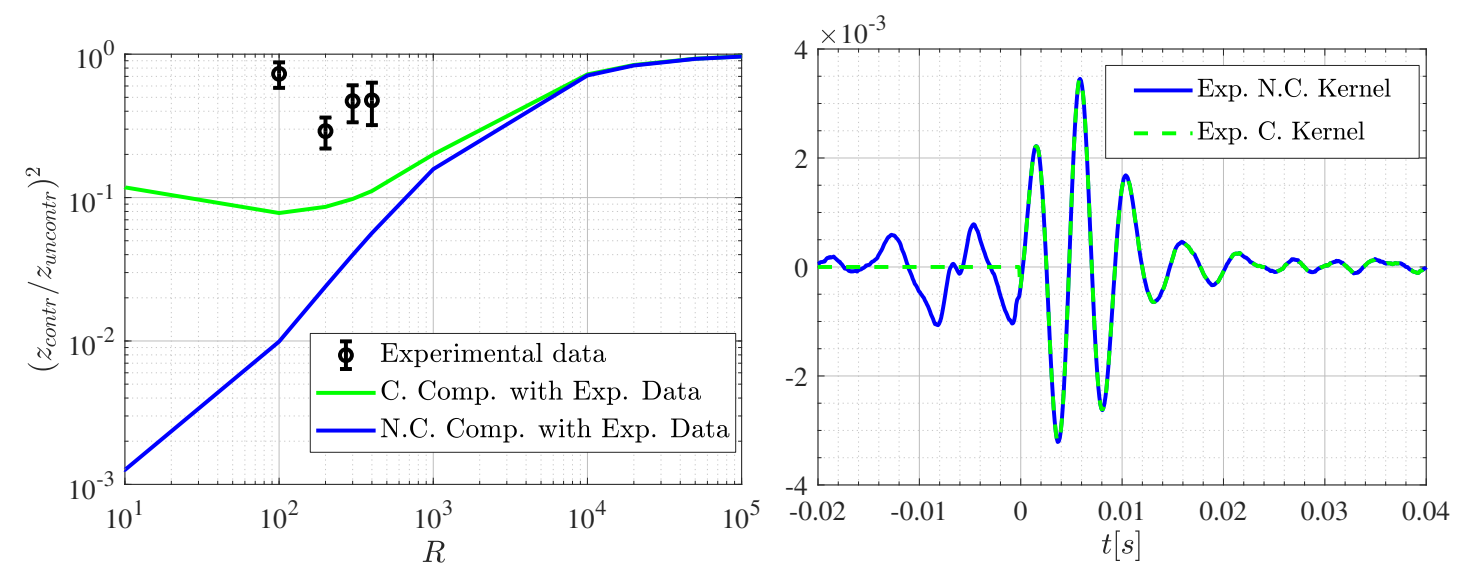

Figure 10: Left: Causal (C.) and non-causal (N.C.) computed performance with experimental data vs. experimental results. Right: Experimental causal (C.) and non-causal (N.C.) kernels for $\mathrm{R}=200$.

signal in the time domain for the $z$ pressure probe.

$$
z_{\text {output }}(t)=G^{y z}(t) * y(t)+K(t) *\left[G^{y u}(t) * y(t)\right]
$$

where stars $*$ denote convolution. Hence, $G^{y z}(t) * y(t)$ is the open-loop behavior and $K(t) *\left[G^{y u}(t) * y(t)\right]$ is the effect of actuation, which tries to act in phase opposition with the open-loop part bringing $z_{\text {output }}$ to a minimum.

It can also be noticed in figure 10 (left) that a large difference in the computed performance is obtained when the non-causal part (N.C.) of the kernel is taken in consideration. Figure 10 (right) shows how significant the non-causal part is in the kernel for the best penalization case when $R=200$. As discussed in section 2.1, real-time applications only allow the use of the causal part of the kernel, and thus the values for negative time delays should be discarded. On the other hand, the offline calculations using equation (8) may include the non-causal part of the kernel in the convolutions. The difference between causal and non-causal performance shows the effect of the negative time delays in the kernel exemplified in figure 10 (right).

The reason of large difference in the computed curves of figure 10 (left) resides at low coherence due to the ambient noise of the wind tunnel, with considerable effect on the $G^{y z}$ transfer function, shown in figure 4. As discussed in section 4.1, such coherence loss at low frequencies makes the experimental transfer function narrower in the frequency domain, which causes a spread in time domain. Therefore the low coherence in the experimental $G^{y z}$ leads to a more significant non-causal part of the kernel, deteriorating the performance of the control.

To confirm this hypothesis, the $G^{y z}$ transfer function was corrupted in the DNS results, which initially had no noise in its signals, to mimic the experimental one. Figure 11 helps to confirm this. The DNS kernels have negligible non-causal part, and the original performances for causal and non-causal control, shown 

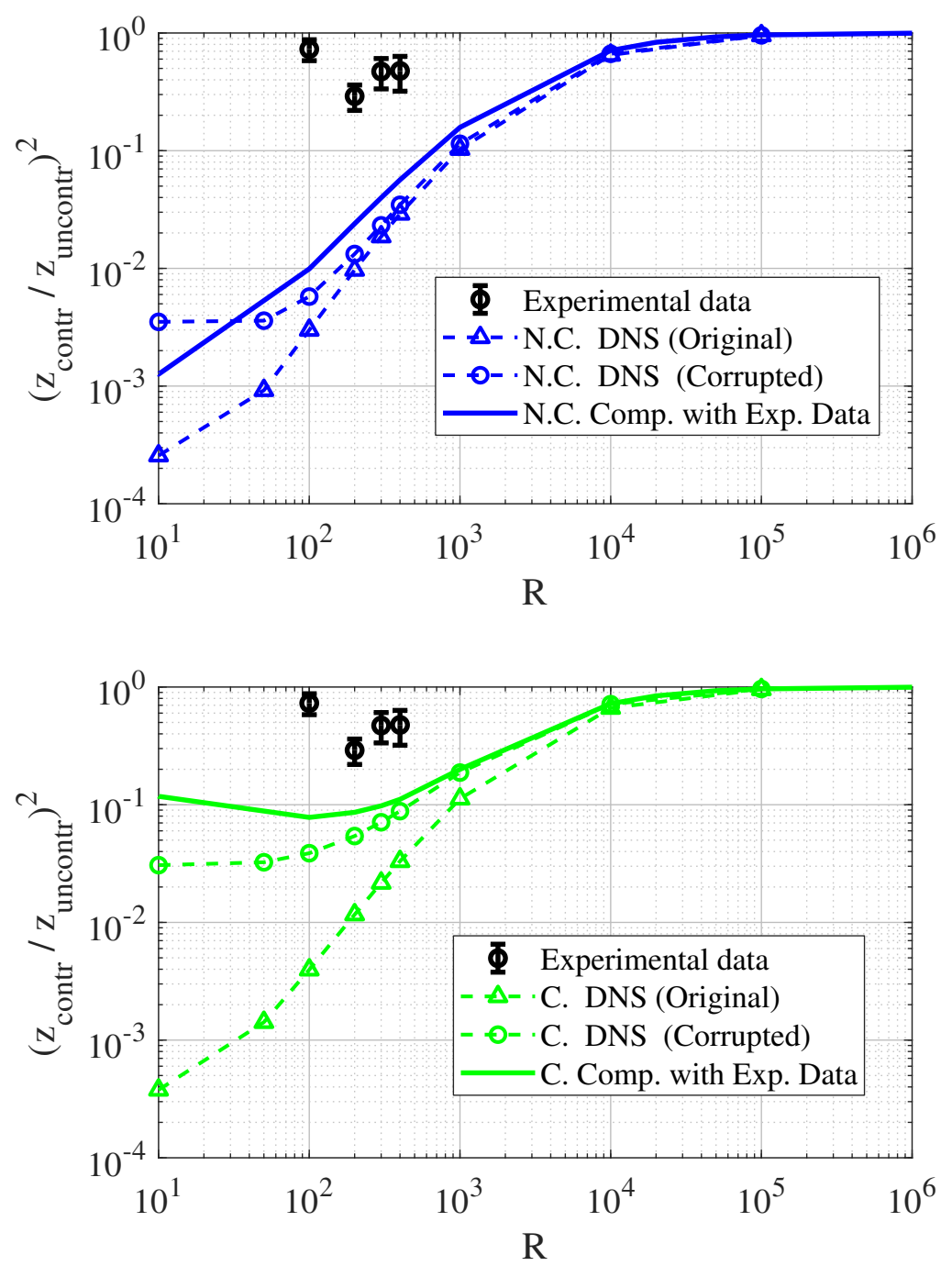

Figure 11: Non-causal (Top) and causal (Bottom) performance using the original DNS data and with the corrupted $G_{y z}$ transfer function.

in figure 11 with triangles, are almost identical and lead to power reductions of up to three orders of magnitude. By corrupting the $G^{y z}$ transfer function in the DNS data to make it similar to the experimental one, the stretching of the kernel in the time domain causes the appearance of a non-causal part, and the performance obtained with the causal part (green line with circles) is close to the one computed with experimental data (continuous line). Notice also that the performance of kernels computed from the corrupted DNS transfer functions now shows significant differences between non-causal (blue) and causal (green) performances, which highlights that the reason to the low- $R$ plateau in performance is the presence of a non-causal part in the kernel. We thus obtain an attenuation close to an order of magnitude in our experiment, limited by the low-frequency noise captured by the microphones. 


\section{Conclusion}

This work is a first experimental attempt of inverse feed-forward control (IFFC) for transition delay through cancellation of Tollmien-Schlichting (TS) waves. IFFC has as advantage the straightforward use of transfer functions, which may be determined directly from experiments, to determine control laws. The IFFC method was shown to be effective, with significant attenuation of TS waves, up to one order of magnitude, even with fluctuations of $\approx 5 \%$ of free-stream velocity in the open circuit wind tunnel.

The experimental results were compared to the direct numerical simulations. Despite encouraging agreement in transfer functions from simulation and experiment, the performance obtained by the DNS was much greater than in the experiments due to the absence of noise. However by corrupting the transfer function to mimic the level of noise in the experiments, the same trend in performance was obtained, showing that simulations are suitable for testing different control techniques for transition delay and giving a reasonable estimation of the expected performance once a representative level of noise is inserted.

The relevant noise in the present experiment is associated with low-frequency acoustic waves present in the test section. Such waves lead to low coherences between TS wave excitation and microphone responses at such low frequencies. This leads to narrower transfer functions in the frequency domain, which in turn become more spread in the time domain, with a non-causal tail appearing for negative time delays. This leads to kernels with non-causal parts. Such effect was confirmed by including a similar low-frequency noise in the simulations, which start to display similar control performance to experiments.

Finally, the present work shows how electret microphones can be used as sensors for closed-loop control of transitional boundary layers. Their relative low cost opens new possibilities for the application of multiple sensors in experimental implementations of control, which is relevant for complex $3 \mathrm{D}$ disturbances. It should be kept in mind that as such microphones measure disturbances other than TS waves (such as ambient noise), they may lead to less accurate transfer functions which in turn are related to the non-causal part of the kernels observed here. However, despite this issue, significant reductions of TS wave amplitude, close to an order of magnitude, were obtained here, which demonstrates the practical feasibility of the present control methods.

\section{Acknowledgments}

We thank our colleagues from the Kwei Lien Feng laboratory at ITA, especially Eng. Wilson Mendes de Souza who provided insight and expertise that greatly assisted the experiments. The authors acknowledge financial support from CNPq, Embraer and CISB. The authors would also like to thank the VINNOVA Projects PreLaFlowDes II and SWE-DEMO for funding. Direct numerical simulations were performed on resources provided by the Swedish National Infrastructure for Computing (SNIC) at NSC, HPC2N and PDC. 


\section{REFERENCES}

Anderson, B. \& Liu, Y. 1989 Controller Reduction: Concepts and Approaches. IEEE Trans. Autom. Control 34, 802-812.

Bagheri, S., Hepffner, J., Schmid, P. J. \& Henningson, D. S. 2009 InputOutput Analysis and Control Design Applied to a Linear Model of Spatially Developing Flows. Appl. Mech. Rev. 62.

Barbagallo, A., Sipp, D. \& Schmid, P. J. 2009 Closed-loop control of an open cavity flow using reduced order models. J. Fluid Mech. 641, 1-50.

Belson, B. A., Semeraro, O., Rowley, C. W. \& Henningson, D. S. 2013 Feedback control of instabilities in the two-dimensional Blasius boundary layer: The role of sensors and actuators. Phys. Fluids 25 (5), 054106.

Bendat, J. S. \& Piersol, A. G. 2011 Random data: analysis and measurement procedures. John Wiley \& Sons.

Cattafesta, L. N. \& Sheplak, M. 2010 Actuators for Active Flow Control. Ann. Rev. Fluid Mech. 43, 247-272.

Corke, T. C., Enloe, C. L. \& Wilkinson, S. P. 2010 Dielectric Barrier Discharge Plasma Actuators for Flow Control. Ann. Rev. Fluid Mech. 42 (1), 505-529.

Devasia, S. 2002 Should model-based inverse inputs be used as feedforward under plant uncertainty? IEEE Transactions on Automatic Control 47 (11), 1865-1871.

Duchmann, A., Simon, B., Tropea, C. \& Grundmann, S. 2014 Dielectric Barrier Discharge plasma actuators for in-flight transition delay. AIAA J. .

Fabbiane, N., Semeraro, O., Bagheri, S. \& Henningson, D. S. 2014 Adaptive and Model-Based Control Theory Applied to Convectively Unstable Flows. Appl. Mech. Rev. 66 (6), 060801.

Fabbiane, N., Simon, B., Fischer, F., Grundmann, S., Bagheri, S. \& HenningSON, D. S. 2015 On the Role of Adaptivity for Robust Laminar-Flow Control. J. Fluid Mech. 767, R1.

Fischer, P., Kruse, J., Mullen, J., Tufo, H., Lottes, J. \& Kerkemeier, S. 2008 Nek5000: Open source spectral element cfd solver.

Grundmann, S., Barckmann, K., Maden, I., Kriegseis, J., Jakirlic, S. Z., Tropea, C. D. \& Grundmann, S. 2014 Evaluating Force Field induced by a Plasma Actuator using the Reynolds-Averaged Navier Stokes Equation. In 52nd Aerospace Sciences Meeting. AIAA.

Grundmann, S. \& Tropea, C. 2008 Active cancellation of artificially Introduced Tollmien-Schlichting waves using plasma actuators. Exp. Fluids 44 (5), 795-806.

GAD-EL HAK, M. 2000 Flow control: passive, active, and reactive flow management. Cambridge University Press.

Hanson, R. E., Bade, K. M., Belson, B. A., Lavoie, P., Naguib, A. M. \& Rowley, C. W. 2014 Feedback control of slowly-varying transient growth by an array of plasma actuators. Physics of Fluids 26 (2), 024102.

Högberg, M., Bewley, T. R. \& Henningson, D. S. 2003 Linear Feedback Control and Estimation of Transition in Plane Channel Flow. J. Fluid Mech. 481, 149-175.

Ilak, M. \& Rowley, C. W. 2008 Modeling of Transitional Channel Flow Using Balanced Proper Orthogonal Decomposition. Phys. Fluids 20, 034103.

Jolibois, J. \& Moreau, E. 2009 Enhancement of the Electromechanical Performances of a Single Dielectric Barrier Discharge Actuator. IEEE Transactions on Dielectrics and Electrical Insulation 16 (3), 758-767. 
JUANG, J. N. \& PAPPA, R. S. 1985 An eigensystem realization algorithm for modal parameter identification and model reduction. J. Guid., Con. Dyn. 8 (5), 620-627.

Kotsonis, M., Giepman, R., Hulshoff, S. \& Veldhuis, L. 2013 Numerical study of the control of tollmien-schlichting waves using plasma actuators. AIAA J. 51 (10), 2353-2364.

Kotsonis, M., Shukla, R. K. \& Pröbsting, S. 2015 Control of Natural TollmienSchlichting Waves using Dielectric Barrier Discharge Plasma Actuators. Intl J. of Flow Control 7 (1-2), 37-54.

Kriegseis, J. 2011 Performance Characterization and Quantification of Dielectric Barrier Discharge Plasma Actuators. PhD thesis, TU Darmstadt.

Kriegseis, J., Möller, B., Grundmann, S. \& Tropea, C. 2011 Capacitance and power consumption quantification of dielectric barrier discharge (DBD) plasma actuators. Journal of Electrostatics 69 (4), 302-312.

Kriegseis, J., Schwarz, C., Tropea, C. \& Grundmann, S. 2013 VelocityInformation-Based Force-Term Estimation of Dielectric-Barrier Discharge Plasma Actuators. J. Phys. D 46 (5), 055202.

Kriegseis, J., Simon, B. \& Grundmann, S. 2016 Towards in-flight application? A review on dielectric barrier discharge-based boundary-layer control. Appl. Mech. Rev. 68 (2).

Kurz, A., Goldin, N., King, R., Tropea, C. D. \& Grundmann, S. 2013 Hybrid Transition Control Approach for Plasma Actuators. Exp. Fluids 54 (11), 1-4.

Kurz, A., Simon, B., Tropea, C. \& Grundmann, S. 2014 Active wave cancelation using plasma actuators in flight. In 52nd Aerospace Sciences Meeting. AIAA.

Laurien, E. \& Kleiser, L. 1989 Numerical simulation of boundary-layer transition and transition control. Journal of Fluid Mechanics 199, 403-440.

Lewis, F. L. \& Syrmos, L. V. 1995 Optimal Control. John Wiley \& Sons, New York.

Liepmann, H. \& NosenchuCK, D. 1982 Active control of laminar-turbulent transition. J. Fluid Mech. 118, 201-204.

LJUng, L. 1999 System identification. Wiley Online Library.

Ma, Z., Ahuja, S. \& Rowley, C. W. 2011 Reduced-order models for control of fluids using the eigensystem realization algorithm. Theoretical and Computational Fluid Dynamics 25 (1-4), 233-247.

Milling, R. W. 1981 Tollmien-Schlichting wave cancellation. Phys. Fluids 24, 979.

Morra, P., Sasaki, K., Hanifi, A., Cavalieri, A. G. V. \& Henningson, D. S. 2020 A realizable data-driven approach to delay bypass transition with control theory. J. Fluid Mech. 883, A33.

Rocklin, G. T., Crowley, J. \& Vold, H. 1985 A comparison of h1, h2, and hv frequency response functions. In Proceedings of the 3rd International Modal Analysis Conference, pp. 272-278. SEM.

Rowley, C. W. 2005 Model reduction for fluids, using balanced proper orthogonal decomposition. Intl J. of Bifurcation and Chaos 15 (03), 997-1013.

Rowley, C. W. \& Dawson, S. T. M. 2017 Model reduction for flow analysis and control. Ann. Rev. Fluid Mech. 49, 387-417.

Sasaki, K., Morra, P., Cavalieri, A., Hanifi, A. \& Henningson, D. 2020 On the role of actuation for the control of streaky structures in boundary layers. $J$. Fluid Mech. 883, A34.

Sasaki, K., Morra, P., Fabbiane, N., Cavalieri, A. G. V., Hanifi, A. \& 
HenNingson, D. S. 2018a On the wave-cancelling nature of boundary layer flow control. Theoretical and Computational Fluid Mechanics 32, 593-616.

Sasaki, K., Tissot, G., Cavalieri, A. V. G., Silvestre, F. J., Jordan, P. \& BIAU, D. 2018b Closed-loop control of a free shear flow: a framework using the parabolised stability equations. Theoretical and Computational Fluid Dynamics 32 (6), 765-788.

Schmid, P. J. \& Henningson, D. S. 2001 Stability and Transition in Shear Flows. Applied Mathematical Sciences v. 142. Springer-Verlag.

Schmid, P. J. \& Sipp, D. 2016 Linear control of oscillator and amplifier flows. Phys. Rev. Fluids 1 (4).

Semeraro, O., Bagheri, S., Brandt, L. \& Henningson, D. S. 2013 Transition delay in a boundary layer flow using active control. J. Fluid Mech. 731 (9), 288-311.

Sheplak, M., Cattafesta, L. \& Nishida, T. 2004 MEMS Shear Stress Sensors: Promise and Progress. 24th AIAA Aerodynamic Measurement Technology and Ground Testing Conference.

Simon, B., Fabbiane, N., Nemitz, T., Bagheri, S., Henningson, D. S. \& Grundmann, S. 2016 In-flight active wave cancelation with delayed-x-lms control algorithm in a laminar boundary layer. Experiments in Fluids 57 (10), 160.

Simon, B., Nemitz, T., Rohlfing, J., Fischer, F., Mayer, D. \& Grundmann, S. 2015 Active flow control of laminar boundary layers for variable flow conditions. Intl J. Heat and Fluid Flow 56, 344-354.

Sipp, D., Marquet, O., Meliga, P. \& Barbagallo, A. 2010 Dynamics and Control of Global Instabilities in Open-Flows: a Linearized Approach. Appl. Mech. Rev. 63 (3), 30801.

Sipp, D. \& Schmid, P. J. 2016 Linear Closed-Loop Control of Fluid Instabilities and Noise-Induced Perturbations: A Review of Approaches and Tools. Appl. Mech. Rev. 68 (2), 020801.

Stoica, P. \& Moses, R. 2007 Spectral analysis of signals. Prentice-Hall.

Sturzebecher, D. \& Nitsche, W. 2003 Active cancellation of Tollmien-Schlichting instabilities on a wing using multi-channel sensor actuator systems . Intl J. Heat and Fluid Flow 24, 572-583.

Thomas, A. S. W. 1983 The control of boundary-layer transition using a wavesuperposition principle. Journal of Fluid Mechanics 137, 233-250.

Wang, J., Choi, K., Feng, L., Jukes, T. \& Whalley, R. 2013 Recent developments in dbd plasma flow control. Prog Aerosp Sci 62, 52-78. 